\title{
Nudos críticos en la inserción de docentes principiantes: lecciones para los programas de formación de profesores
}

\author{
Gerardo I. Sánchez ${ }^{(1,2)}$, Ximena E. Jara (2) y Fernando A. Verdugo (2) \\ (1) Centro de Investigación en Educación para la Justicia Social Universidad Católica del Maule (CIEJUS), Talca - Chile. \\ (2) Universidad Autónoma de Chile, Pedagogía en Historia, Geografía y Ciencias Sociales y Pedagogía en Inglés, Talca, \\ Chile. (correo-e: gsanchez@ucm.cl; xjaraa@autonoma.cl; fverdugop@uautonoma.cl)
}

Recibido Ene. 27, 2020; Aceptado Mar. 30, 2020; Versión final May.15, 2020, Publicado Ago. 2020

\begin{abstract}
Resumen
Este artículo problematiza en los nudos críticos del profesorado principiante en el contexto de la Ley de Desarrollo Profesional Docente en Chile y un nuevo orden social y cultural que complejiza la tarea docente en términos de sus demandas. Mediante una aproximación cualitativa con entrevistas semiestructuradas a profesores de educación primaria, especial e infantil se determinaron las dificultades en cuatro ámbitos de acción que definen una buena docencia. El análisis permite identificar preocupaciones asociadas a la creación de un ambiente propicio de aprendizaje, a responsabilidades profesionales, a una enseñanza para el aprendizaje de todos los estudiantes, y la preparación de la misma. Se concluye que el principiante enfrenta su trabajo con esquemas de actuación fragmentados respecto a las situaciones que experimenta, razón por la cual la formación del profesorado debe contemplar la sensibilidad cultural y mayor vínculo con los territorios escolares de inserción.
\end{abstract}

Palabras clave: profesorado principiante; inserción profesional; preocupaciones; tarea docente

\section{Critical knots in the insertion of beginner teachers: lessons for teacher training programs}

\begin{abstract}
This research article problematizes the critical knots of beginner teachers in the context of the Teaching Professional Development Act in Chile and a new social and cultural order that complicates the teaching task in terms of its demands. A qualitative approach with semi-structured interviews with primary and special child education teachers identified the difficulties in four areas of action that define good teaching. The analysis allows to identifying concerns associated with the creation of an environment conducive to learning, to professional responsibilities, to teaching for the learning of all students, and its preparation. It is concluded that the beginner faces her/his work with fragmented action schemes regarding the situations she/he experiences, which is why the training of teachers should consider cultural sensitivity and a greater link with the school insertion territories.
\end{abstract}

Keywords: beginner teachers; professional insertion; concerns; teaching task 


\section{INTRODUCCIÓN}

En la mayoría de los países occidentales, los sistemas escolares se encuentran ante exigencias, expectativas y demandas sin precedentes; situación que repercute con más fuerza en el profesorado (Bottery, 2006). Comprender cómo se forman los docentes y qué ocurre a lo largo de su etapa profesional es un desafío impostergable para dimensionar y favorecer su desarrollo profesional, redescubriendo la nueva fisonomía de su rol. En ese escenario, la inserción profesional del profesorado novel y, en consecuencia, el vínculo entre la formación inicial y continua constituye un componente de calidad de primer orden del sistema educativo, lo que se traduce en la exigencia de un profesorado bien formado, capaz de ofrecer respuestas innovadoras y dotado de herramientas para comprometerse con los territorios escolares y su transformación (Goh et al., 2017, Alsaeedi y Male, 2013). La complejidad que adquiere en la actualidad la tarea docente exige un cambio de enfoque, más aún cuando la evidencia que ofrecen los estudios sobre la vida de los profesores y su inserción profesional concluye que estos no han cumplido la etapa de formación al salir de una institución universitaria. En verdad, recién al comenzar su ejercicio profesional entran en una etapa de aprendizaje docente que es clave para lo que será su futuro ejercicio. Desde la perspectiva de Ávalos y Valenzuela (2016), Çakmak et al. (2019), este aprendizaje de la profesión en el caso del principiante ha sido tradicionalmente un aprendizaje solitario sin ayudas especiales, y, por tanto, centralmente, un aprendizaje de la experiencia.

Estudios internacionales y nacionales ponen de manifiesto la preocupación por la problemática de la inserción profesional y las complejidades que enfrenta el poder avanzar hacia enfoques de trabajo más innovadores que logren tomar distancia de las visiones tradicionales presentes en el ejercicio de la docencia. Esta inserción - articulada en torno a dudas, inseguridades y ansiedad que se acumulan y conviven no siempre de manera armónica en la práctica-, es una etapa en que se viven dificultades desde los propios recursos o con apoyos externos (Alliaud, 2017); en que se enseña y se debe aprender a enseñar (Hogan et al., 2010, FeimanNemser, 2001); una fase que requiere superar el trabajo aislado y disponer de condiciones orientadas a la colaboración.

Los estudios en Chile (Ávalos, 2009) confirman que los docentes principiantes tienen las mismas dificultades que los de otros países, no obstante, en el caso nacional estas se abordan más desde el ensayo y el error pues no se dispone de ayuda formal al respecto. Más específicamente, el estudio de Cisternas (2011) confirma que la inserción está condicionada por las oportunidades que la formación inicial brindó para problematizar la realidad más allá de los espacios de práctica, la cultura de los establecimientos de inserción y las características personales del docente novel. Finalmente, también se constata que el contexto limita la práctica pedagógica y lleva a acatar prescripciones orientadas al orden y el silencio. "El enfoque donde el estudiante y el aprendizaje activo son el centro, aparece como difícil y lento de implementar en la escuela, donde la presión por obtener cobertura curricular y por los resultados en mediciones estandarizadas legitiman una tradición pedagógica" (Ruffinelli et al., 2017, p. 381). La inserción profesional enfrenta al novel a problemáticas diversas derivadas de "las nuevas relaciones interpersonales que deben establecer, del escaso conocimiento de la cultura escolar o de las múltiples tareas que deben asumir en forma simultánea, esto sumado a que deben asumir su rol de enseñantes para el cual fueron formados" (Solís et al., 2016, p. 332)

Este profesorado novel experimenta un momento crucial en su trayectoria docente pues debe adquirir un adecuado conocimiento y competencia profesional en un tiempo en general breve, movilizando en ello una serie de preocupaciones que hacen referencia a las condiciones laborales y cómo se organiza el trabajo docente, en términos de lo que significa la utilización de tiempos, estilos de trabajo, soportes técnicopedagógicos y condiciones de aula, así como también las condicionantes derivadas de un perfil claramente diverso de los estudiantes, de sus familias y los contextos socioculturales de origen (Chaaban y Du, 2017).

La llegada, por primera vez, de un joven profesor o profesora a una escuela se expresa en formas diversas cuyo denominador común es que en todos los casos se trata de un verdadero "reality shock" es decir una "sacudida de realidad", "de transición", "de práctica" o un "reinwasheffekt" o efecto de purificación (Tardif, 2004, Fantilli y McDougall, 2009). Este golpe de realidad da cabida a un proceso de aprendizaje de la profesión que transita desde preocupaciones centradas en su propia persona: “¿cómo podré hacerlo?" “¿seré capaz de..."? hasta preocupaciones que se dirigen a sus alumnos, sea en términos de su comportamiento o su aprendizaje, del tipo: ¿cómo asegurar que mis alumnos se comporten y muestren disciplina? ¿cómo enseñar este tema para que lo entiendan mejor?", "¿están aprendiendo lo que necesitan aprender"? (Kim y Cho, 2014)

Esta inserción del principiante al trabajo docente en tanto proceso de socialización, orientado a asumir un rol en la institución se resuelve en las interacciones entre la experiencia biográfica, la formación inicial recibida y los factores contextuales y se traduce en "choque cultural" (Amin y Rahimi, 2018, Pardo et al., 2019) el que desde el planteamiento de Tardif (2004) implica la asimilación de una realidad compleja, que se presenta todos los días, particularmente, en el momento en que se está comenzando a asumir sus funciones en la enseñanza. 
En esa perspectiva, al entender que la inserción a la docencia involucra relaciones multidimensionales entre satisfacción, motivación y expectativas desde las cuales se resuelve el trabajo docente, diversos sistemas educacionales formulan estándares o criterios que definen lo que debe saber y poder ejecutar un docente que egresa de una institución formadora, como ocurre en Canadá, el Reino Unido, Estados Unidos, Australia y Nueva Zelanda. Hay diferencias entre las distintas formulaciones respecto a cómo se define el trabajo docente y qué se desea evaluar, y ellas generalmente, se expresan en una mayor o menor especificación de competencias observables y evaluables.

Chile, ha optado por una formulación de estándares nacionales que orientan el trabajo de las instituciones formadoras, como también marcos reguladores para los profesores en ejercicio. En nuestro país, las expectativas sobre la calidad del desempeño profesional, se manifiestan en cuatro dimensiones principales que dan origen al Marco para la Buena Enseñanza: la preparación (o momento preactivo); la creación de un ambiente propicio para la enseñanza; la enseñanza propiamente tal (momentos interactivos desarrollados en el aula); y las acciones que ocurren después de la enseñanza o fuera del aula y que se relacionan con el comportamiento profesional del docente. Cada una de estas dimensiones integra un conjunto de indicadores que sirven de guía tanto para la evaluación del desempeño del futuro docente, como para orientar la calidad de las experiencias educativas que se les ofrece en el desarrollo de actividades de inducción.

En el contexto nacional, tras entender que las experiencias de iniciación de los docentes no se explican sólo por lo que acontece en el aula con los estudiantes, sino también y fundamentalmente por las políticas educativas en torno a la profesión docente, el currículo y sus orientaciones, los sistemas de control y rendimiento de cuentas y los procesos de segregación escolar en términos de desigualdades sociales, económicas y espaciales; se define la implementación en el año 2016, de una Ley de Desarrollo Profesional Docente que visibiliza la etapa de la inducción y sus requerimientos, pues involucra para el profesorado principiante un aprendizaje, práctica y responsabilidad profesional; facilitando su inserción en el desempeño profesional y en la comunidad educativa a la cual se integra.

A lo anterior se agrega, la existencia de nuevos ordenes sociales y culturales que vienen a complejizar el escenario de ejercicio de la docencia. Las condiciones en las que se desarrollan los procesos de educación en la actualidad han cambiado, "son otros los chicos, los jóvenes, los adultos, las relaciones entre generaciones, el peso y la legitimidad de las instituciones, los vínculos con el conocimiento, etc" (Alliaud, 2017, p.22); razón por la cual resulta novedoso problematizar en las preocupaciones del profesorado principiante intentando ampliar el foco tradicional de atención del aula al del contexto escolar (pares) y comunitario (familia), entender mejor las relaciones dinámicas entre el principiante y el contexto escolar; y en consecuencia, el vínculo entre universidad y centros educacionales, procurando así, fortalecer en términos bidireccionales los programas de formación de profesores y el ejercicio profesional.

En el marco del método de indagación y de acuerdo con las características del objeto de estudio que se presenta se optó por una estrategia metodológica cualitativa, ya que uno de los propósitos de este estudio es la comprensión de la realidad desde la perspectiva de los sujetos (Flick, 2007). Busca, conocer las orientaciones subjetivas de profesores principiantes, a través de la técnica de entrevista, como forma de reconstruir la experiencia de integración que vive en sus primeros años de inserción laboral a la docencia. El resultado obtenido permite entender las preocupaciones de la docencia más allá del espacio del aula, y fuertemente tensionadas por los nuevos órdenes sociales y culturales, que encuentran su expresión en demandas de diversidad, trabajo con la familia y requerimientos asociados a cultura escolar.

Se pone de manifiesto, la necesidad de una formación de profesores más vinculada a las necesidades de los contextos escolares, y una inserción a la docencia que se concibe como problemática y desafiante, y que requiere acompañamiento formal pues institucional y biográficamente en ella se pone en juego el proceso de integración social que acopla expectativas y aspiraciones con las estructuras educacionales y, por tanto, laborales.

\section{METODOLOGÍA}

Este estudio se enmarca dentro de un enfoque de investigación cualitativa, de tipo hermenéutica y tiene la intención de discutir, desde las preocupaciones que experimenta el profesorado novel o principiante, las complejidades que enfrenta la inserción a la docencia, sus nudos críticos y las demandas que se derivan a los programas de formación de profesores. A partir de un muestreo de tipo intencionado, se trabajó con 20 profesores de educación parvularia, educación especial y educación básica de la región del Maule quienes ofrecieron sus experiencias en torno al proceso de inserción a la docencia. De este grupo, 15 corresponden a mujeres y 05 varones, con edades entre los 25 y 30 años. Los criterios para elegirles fueron: 1) Tener una experiencia laboral que se encuentre comprendida entre 1 y 3 años; 2) Encontrarse trabajando en el momento de la investigación; y 3) Aceptaran participar de forma voluntaria, tras firma de consentimiento. 
Se optó por un abordaje de tipo cualitativo, mediante un diseño de investigación de tipo transeccional no experimental con un nivel de profundidad descriptivo. Contempló una aproximación de carácter intensivo mediante el uso de una entrevista semiestructurada (Flick, 2007) a los profesores principiantes, la cual permitió indagar en torno a las diversas preocupaciones y dificultades experimentadas en su inserción a la docencia. Esta aproximación metodológica, con guión de preguntas previamente validado, permitió acceder a datos descriptivos y a un abordaje interpretativo de los sujetos investigados.

Las demandas, dificultades o necesidades consultadas a los profesores principiantes se entienden como las preocupaciones que los docentes informan, como difíciles de ejecutar o resolver en la interacción con los escolares, profesores, directivos y familia de los cursos donde desarrollan sus prácticas. Para contextualizar las preocupaciones se considera como referente el Marco de la Buena Enseñanza (MBE), instrumento oficial y vigente para la evaluación docente y para orientar las prácticas pedagógicas de los profesores chilenos. Este MBE, permite establecer un recorrido completo del acto pedagógico, otorgando un papel integral al docente desde la preparación de la clase, hasta la reflexión de sus decisiones pedagógicas y profesionales. Este instrumento busca ser un eje articulador de las prácticas efectivas en el sistema escolar y movilizador del mejoramiento y desarrollo profesional. Las variables del instrumento fueron establecidas a partir de los 4 dominios del MBE: 1) Preparación para la enseñanza, 2) Creación de un ambiente propicio para el aprendizaje, 3) Enseñanza para el aprendizaje de todos los estudiantes y 4) Responsabilidades profesionales. Estas variables representan lo que los docentes deben saber y saber hacer, desde el vínculo empático que requiere la tarea de educar y la compleja diversidad cultural donde se produce el acto educativo.

Se utilizó análisis cualitativo de la información (Ruiz, 2009). A partir de la codificación de las entrevistas se formularon categorías inductivas, acompañadas de la evidencia empírica correspondiente, desde la cual se realizó la discusión de la información y obtención de conclusiones. Para llevar a cabo la sistematización de la información, la Tabla 1 registra la frecuencia de preocupaciones que los profesores principiantes declararon por cada una de las dimensiones y categorías, lo que permite apreciar la densidad de la información, y en definitiva de los relatos:

Tabla 1: frecuencia de preocupaciones por dimensión y categorías

\begin{tabular}{|c|c|c|c|}
\hline Dimensión & Frecuencia & Categoría & Frecuencia \\
\hline \multirow[t]{3}{*}{ Dimensión Preparación de la enseñanza } & \multirow[t]{3}{*}{30} & Conocimiento de sus estudiantes & 13 \\
\hline & & Dominio de la didáctica de las disciplinas & 10 \\
\hline & & Estrategias de evaluación & 7 \\
\hline \multirow[t]{3}{*}{$\begin{array}{l}\text { Creación de un clima propicio para el } \\
\text { aprendizaje }\end{array}$} & \multirow[t]{3}{*}{31} & $\begin{array}{l}\text { Establecimiento y mantención de normas consistentes de } \\
\text { convivencia }\end{array}$ & 14 \\
\hline & & Manifestación de expectativas & 10 \\
\hline & & Establecimiento de ambiente organizado de trabajo & 7 \\
\hline \multirow[t]{3}{*}{$\begin{array}{l}\text { Enseñanza para el Aprendizaje de todos los } \\
\text { Estudiantes }\end{array}$} & \multirow[t]{3}{*}{35} & $\begin{array}{l}\text { Estrategias de enseñanza desafiantes, coherentes y } \\
\text { significativas }\end{array}$ & 13 \\
\hline & & Optimización del tiempo para la enseñanza & 10 \\
\hline & & $\begin{array}{l}\text { Evaluación y monitoreo para la comprensión y apropiación de } \\
\text { los contenidos }\end{array}$ & 12 \\
\hline \multirow[t]{4}{*}{ Responsabilidades Profesionales } & \multirow[t]{4}{*}{45} & Reflexión sistemática sobre su práctica & 7 \\
\hline & & $\begin{array}{l}\text { Construcción de relaciones profesionales y de equipo con sus } \\
\text { colegas }\end{array}$ & 10 \\
\hline & & Relaciones de colaboración con los padres y apoderados & 14 \\
\hline & & $\begin{array}{l}\text { Manejo actualizado de la profesión, el sistema educativo y las } \\
\text { políticas vigentes }\end{array}$ & 14 \\
\hline
\end{tabular}

\section{RESULTADOS}

Los resultados se presentan organizados en las dimensiones del marco para la buena enseñanza: Preparación de la enseñanza, Creación de un ambiente propicio para el aprendizaje, Enseñanza para el aprendizaje de todos los estudiantes y Responsabilidades profesionales. En cada una de las dimensiones que se presentan a continuación, se identifica las categorías de análisis levantadas a partir de las entrevistas realizadas acompañadas de extractos de registros de habla.

\section{Preparación de la Enseñanza}

Este ámbito que busca disponer de un diseño curricular de aula efectivo para lograr equidad y oportunidad de aprendizaje de todos los estudiantes, considera como primordial, conocer en profundidad el tipo de estudiante que se atiende para poder trazar experiencias de aprendizaje ajustadas a las propias necesidades 
del contexto, que utilice la variabilidad de sus estudiantes como fuente de enriquecimiento y posibilidades de innovar la enseñanza, superando la relación de enseñanza unidireccional donde el aprendizaje es solo responsabilidad del estudiante que lo recibe. En relación a la preparación de la enseñanza, la Tabla 2 registra las preocupaciones que los profesores principiantes declaran:

Tabla 2: preocupaciones asociadas a la dimensión preparación de la enseñanza

\begin{tabular}{|l|l|}
\hline Categorías de análisis & Extractos de registros de datos \\
\hline $\begin{array}{l}\text { Conocimiento de sus } \\
\text { estudiantes }\end{array}$ & $\begin{array}{l}\text { "Desconozco las particularidades familiares y culturales de mis alumnos, lo que dificulta la planificación y el } \\
\text { desarrollo de la docencia" (E2). } \\
\text { "Preocupación por atender las particularidades de mis estudiantes (en relación a la familia, su cultura e intereses) y } \\
\text { sus diferentes formas de aprender; es decir ritmos y estilos de aprendizaje" (E12). } \\
\text { "Inseguridad y escaso conocimiento respecto a las necesidades e intereses que presentan sus estudiantes en el } \\
\text { aula" (E5) }\end{array}$ \\
\hline $\begin{array}{l}\text { Dominio de la didáctica } \\
\text { de las disciplinas }\end{array}$ & $\begin{array}{l}\text { "Si bien considero que domino la disciplina que enseño como docente de educación especial; sin embargo, al ser } \\
\text { una disciplina transversal, tengo dificultad para seleccionar las estrategias de enseñanza adecuadas y que permitan } \\
\text { la vinculación con los contenidos de otras asignaturas" (E6). } \\
\text { "Lo más complejo ha sido el lograr pensar y diseñar estrategias que me permitan abordar los distintos ritmos y } \\
\text { estilos de aprendizaje de mis alumnos" (E20). } \\
\text { "Dificultades en la elaboración de material diversificado para los estudiantes, debido al escaso conocimiento de las } \\
\text { características de ellos" (E16). }\end{array}$ \\
\hline $\begin{array}{l}\text { Estrategias de } \\
\text { evaluación }\end{array}$ & $\begin{array}{l}\text { "Una cuestión que me ha complicado es la elaboración de instrumentos de evaluación que se adapten a las distintas } \\
\text { "No manejo mucha variedad de formas de evaluación, por lo tanto, no logro visualizar con certeza y tranquilidad } \\
\text { para mí, en qué medida los y las estudiantes progresan" (E11). } \\
\text { "Siento inseguridad con la evaluación, no me siento segura que lo estoy haciendo bien" (E3). }\end{array}$ \\
\hline
\end{tabular}

En esta dimensión, los principales nudos problemáticos se relacionan con el conocimiento de las características y experiencias personales, familiares y culturales de sus estudiantes a la hora de planificar la enseñanza; así como también, y en estrecha relación con ello, el manejo de la didáctica de las diversas disciplinas que ha de enseñar. Específicamente, las dificultades se plantean en términos de cómo representar esos contenidos a enseñar en una forma tal que puedan ser entendidos por alumnos que se reconocen diversos, lo que obliga a examinar el repertorio de técnicas instruccionales que tiene a disposición y seleccionar las más adecuadas. Se confirma que el perfil diverso de los estudiantes, de sus familias y sus contextos socioculturales de origen (Chaaban y Du, 2017) genera fuente de tensión al trabajo del docente principiante. Asimismo, también emergen dificultades referidas a las estrategias de evaluación utilizadas, y particularmente que dichas estrategias ofrezcan a los estudiantes oportunidades equitativas para demostrar lo que han aprendido.

\section{Creación de un clima propicio para el aprendizaje}

La clase entendida como ecosistema es el ambiente interno y comunicativo que vincula a alumnos, docentes y contenidos, enmarcados en coordenadas espaciales, temporales y socioculturales. La tarea en este ámbito se asocia con decidir ciertas estrategias o acciones que faciliten un espacio de aprendizaje afectivo, seguro, motivante y que atienda la diversidad de características e intereses de los estudiantes, por lo tanto, el aula ya no puede ser entendida como un espacio de transmisión de conocimientos, sino más bien un espacio de construcción colectiva para el aprendizaje. En relación a la creación de un ambiente propicio para el aprendizaje, la tabla 3 muestra las preocupaciones de los profesores principiantes en los términos siguientes:

Los relatos de los participantes del estudio ponen de manifiesto preocupaciones centradas en la habilidad para establecer y mantener normas consistentes de convivencia en el aula. Específicamente el asegurar conocimiento y comprensión de las normas por parte de sus alumnos; su congruencia con las necesidades de la enseñanza y una convivencia armónica: y el movilizar respuestas asertivas - expresadas en decisionesfrente al quiebre de las normas también constituye una dificultad ampliamente reconocida en los principiantes. Un ámbito que se muestra sensiblemente preocupante para este profesorado se relaciona con el desafío de sostener altas expectativas sobre las posibilidades de aprendizaje y desarrollo de todos sus alumnos, lo que se traduce en dos nudos críticos. Por una parte, presentar situaciones de aprendizaje de manera interesante y adecuada a la edad e intereses de sus estudiantes, otorgando el tiempo suficiente. Y por otra, favorecer el desarrollo de la autonomía de los alumnos en situaciones de aprendizaje, estimulando de manera gradual y sistemática la indagación, la formulación de opiniones y el hallazgo de soluciones propias. Los resultados en este ámbito, ponen de manifiesto las dificultades para enganchar a sus estudiantes en una dinámica de protagonismo y enseñanzas activas (Ruffinelli et al., 2017), derivadas además del cambio en el perfil experimentado por el alumnado (Alliaud, 2017). 
Tabla 3: preocupaciones asociadas a la dimensión creación de un ambiente propicio para el aprendizaje

\begin{tabular}{|l|l|}
\hline Categorías de análisis & Extractos de registros de datos \\
\hline $\begin{array}{l}\text { Establecimiento y } \\
\text { mantención de normas } \\
\text { consistentes de } \\
\text { convivencia }\end{array}$ & $\begin{array}{l}\text { "Me ha resultado difíil establecer normas de comportamiento que sean comprensibles por los estudiantes, y la } \\
\text { necesidad de respetarlas" (E3). } \\
\text { "Me ha costado lograr normas de convivencia consensuadas con todos los alumnos" (E9). } \\
\text { "Dificultad para generar respuestas asertivas y efectivas frente al quiebre de las normas de convivencia que suele } \\
\text { ser un problema recurrente el manejo de la disciplina" (E13). } \\
\text { "Muchas veces dudo de mis habilidades para generar ambientes de clases saludables, son escasas mis } \\
\text { estrategias para mantener la atención en la tarea, la resolución pacífica de conflictos, y abordar de manera } \\
\text { formativa las interrupciones fuera de lugar" (E17). }\end{array}$ \\
\hline $\begin{array}{l}\text { Manifestación de } \\
\text { expectativas }\end{array}$ & $\begin{array}{l}\text { "Siento que necesito generar autonomía en mis estudiantes en situaciones de aprendizaje, debido a que buscan } \\
\text { siempre la confirmación en todo lo que hacen" (E1). } \\
\text { "Tengo debilidad a la hora de favorecer la autonomía de mis estudiantes pues todo tienden a preguntarlo y no logro } \\
\text { que los niños trabajen por sí mismos" (E5). } \\
\text { "Dificultad de herramientas para la preparación de actividades desafiantes y creativas para los estudiantes" (E10). } \\
\text { "Dificultad en la clase para ofrecer desafíos cognitivos a mis niños y salirse un poco del trabajo con la guía o el } \\
\text { libro" (E15). }\end{array}$ \\
\hline $\begin{array}{l}\text { Establecimiento de } \\
\text { ambiente organizado de } \\
\text { trabajo }\end{array}$ & $\begin{array}{l}\text { "Dificultad en la manera de utilizar el tiempo, me cuesta observar rutinas establecidas durante el desarrollo de las } \\
\text { "Dificultades para establecer y mantener normas de convivencia, claras y que perduren en el aula, facilitando el } \\
\text { aprendizaje de todos sus estudiantes, suelo perder demasiado tiempo en la disciplina y la clase se me escapa" } \\
\text { (E7). }\end{array}$ \\
\hline
\end{tabular}

\section{Enseñanza para el aprendizaje de todos los/as estudiantes}

Asegurar este ámbito significa que el docente comprenda que no basta impartir una clase frontal para todos los estudiantes, que deposita saberes en educandos que pasivamente recepcionan sin diálogo ni problematización. Por el contrario, entender que el aprendizaje se construye en interacciones y mediaciones dentro del aula que impliquen desafíos, autonomía y conocimiento de cómo los propios estudiantes aprenden. Enfocar las clases desde este dominio implica fomentar el trabajo colaborativo, autónomo y orientado al desarrollo de habilidades asociadas a objetivo de aprendizaje, monitoreadas para los ajustes que sean necesarios dada la diversidad de estudiantes presentes en el aula. En relación a la enseñanza para el aprendizaje de todos los estudiantes, la tabla 4 muestra las preocupaciones de los profesores principiantes en el ámbito en cuestión.

Tabla 4: preocupaciones asociadas a la dimensión enseñanza para el aprendizaje de todos los estudiantes

\begin{tabular}{|c|c|}
\hline Categorías de análisis & Extractos de registros de datos \\
\hline $\begin{array}{l}\text { Estrategias de enseñanza } \\
\text { desafiantes, coherentes y } \\
\text { significativas. }\end{array}$ & $\begin{array}{l}\text { "Uso variadas estrategias de enseñanza, preparo mucho material, sin embargo, no siempre logro el } \\
\text { aprendizaje de todos los estudiantes pues no me resulta fácil compatibilizar los estilos de aprendizajes de } \\
\text { los alumnos y alumnas" (E14). } \\
\text { "Tengo dificultades al momento de propiciar la participación de todos mis estudiantes, focalizándome en } \\
\text { los alumnos que presentan interés, en desmedro de los que suelen distraerse con facilidad" (E9). } \\
\text { "Si bien intento atender a la diversidad de mis estudiantes, me resulta difícil asegurar actividades potentes, } \\
\text { ya que la mayoría de las veces resultan poco desafiantes lo que genera frustración o desinterés" (E2). } \\
\text { "Utilizo diversas actividades, pero estas son monótonas, poco lúdicas y desafiantes para los estudiantes, } \\
\text { no logrando promover por completo el interés y el desarrollo de habilidades superiores" (E6). }\end{array}$ \\
\hline $\begin{array}{l}\text { Optimización del tiempo para la } \\
\text { enseñanza. }\end{array}$ & $\begin{array}{l}\text { "Diseño variadas actividades para consolidar los aprendizajes, pero me cuesta mucho ceñirme a un tiempo } \\
\text { determinado, asegurando un uso efectivo del tiempo" (E4). } \\
\text { "Preparo actividades para todos mis estudiantes, sin embargo, necesito focalizar los estudiantes con } \\
\text { aprendizaje lento y aquellos que van más avanzados para diseñar actividades acordes a su nivel o más } \\
\text { desafiantes en el caso de los alumnos adelantado" (E19). } \\
\text { "Dificultad en utilizar el tiempo en función de los objetivos de la clase y no solo en la disciplina de los niños, } \\
\text { pues ocupo mucho tiempo intentando que se callen y se concentren en el trabajo, se distraen mucho" } \\
\text { (E17). }\end{array}$ \\
\hline $\begin{array}{l}\text { Evaluación y monitoreo para la } \\
\text { comprensión y apropiación de } \\
\text { los contenidos. }\end{array}$ & $\begin{array}{l}\text { "Debilidad en estrategias de retroalimentación, que permiten a los estudiantes tomar conciencia de sus } \\
\text { logros de aprendizaje..." (E5). } \\
\text { "El proceso de retroalimentación en las evaluaciones es muy acotado. Los niños están focalizados en el } \\
\text { hacer y terminar rápido las tareas, pero les cuesta detenerse y pensar en lo que están haciendo, son muy } \\
\text { impulsivos" (E10). } \\
\text { "Desconocimiento de estrategias de retroalimentación para el desarrollo y finalización de las clases lo que } \\
\text { conlleva a que no existe claridad si los estudiantes aprenden o entienden lo que enseña" (E16). }\end{array}$ \\
\hline
\end{tabular}


Específicamente, los relatos de los participantes del estudio ponen de manifiesto nudos problemáticos en este ámbito referidos a la naturaleza de las estrategias, la gestión del tiempo, y el uso pedagógico de la evaluación. En primer lugar, en cuanto a disponer de estrategias de enseñanza desafiante, coherente y significativa para los estudiantes, reconocen dificultad al intentar estructurar situaciones de aprendizajes atendiendo a su diversidad de saberes, intereses y experiencias. En segundo lugar, están las dificultades relacionadas con optimizar el tiempo disponible para la enseñanza, ya sea atendiendo los objetivos de la clase y las necesidades derivadas del ritmo de aprendizaje de sus estudiantes, siendo este último el factor más problemático para el profesorado principiante. En tercer lugar, emergen las dificultades para evaluar y monitorear el proceso de comprensión y apropiación de los contenidos por parte de los estudiantes, lo que se traduce en la utilización de la retroalimentación para ayudar a los alumnos a tomar conciencia de sus logros de aprendizaje. Las preocupaciones en el ámbito están representadas por las dificultades para concretar un proceso educativo con centralidad en el aprendizaje, derivado de la existencia de un perfil diverso de estudiante que tensiona el desarrollo de las actividades, además de la presencia de una cultura escolar y modelos instalados, que terminan por dotar de complejidad a la tarea docente (Ferrada, 2019; Tardif, 2004).

\section{Compromiso con el desarrollo profesional}

Dentro de los 4 dominios del MBE, el eje vertebral que moviliza las prácticas efectivas de los docentes está relacionado con el compromiso de los docentes en los aprendizajes de los estudiantes. Evaluar el efecto de su práctica en los estudiantes, involucrarse con sus pares y reconocer sus propias necesidades, es fundamental para su aprendizaje profesional. En relación al compromiso con el desarrollo profesional, la Tabla 5 identifica las preocupaciones de los profesores principiantes en los términos siguientes.

Tabla 5: preocupaciones asociadas a la dimensión responsabilidades profesionales

\begin{tabular}{|c|c|}
\hline Categorías de análisis & Extractos de registros de datos \\
\hline Reflexión sistemática sobre su práctica & $\begin{array}{l}\text { "Me resulta difícil hacer reflexión sobre mi trabajo, pues no es fácil equilibrar los tiempos frente a la } \\
\text { carga administrativa que encierra la docencia" (E3). } \\
\text { "No es fácil empoderarse del rol, cuesta tomar decisiones en el aula" (E6). } \\
\text { "Noto que me cuesta el análisis crítico de las fortalezas y debilidades de mis prácticas, con relación } \\
\text { a sus efectos sobre los aprendizajes de los estudiantes para reformularlas y hacerlas más efectivas } \\
\text { y pertinentes" (E15). }\end{array}$ \\
\hline $\begin{array}{l}\text { Construcción de relaciones } \\
\text { profesionales y de equipo con sus } \\
\text { colegas. }\end{array}$ & $\begin{array}{l}\text { "Observo cierto desinterés para dialogar con los pares de nivel en torno a aspectos pedagógicos lo } \\
\text { que impide la reflexión colaborativa" (E7). } \\
\text { "No es fácil integrarse con los demás profesores, hay mucha desconfianza e individualismo" (E13). } \\
\text { "He notado dificultades para involucrarme en diálogos con mis pares respecto a prácticas } \\
\text { pedagógicas y su diseño" (E1). } \\
\text { "Mucho trabajo administrativo disminuye el tiempo para el encuentro y reflexión con otros, aunque } \\
\text { no hay mucha disposición de los demás a colaborar o prestar ayuda" (E19). }\end{array}$ \\
\hline $\begin{array}{l}\text { Relaciones de colaboración cor } \\
\text { padres y apoderados. }\end{array}$ & $\begin{array}{l}\text { "Aumentar estrategias de cómo involucrar a las familias en actividades de aprendizaje, recreación y } \\
\text { convivencia de sus alumnos" (20). } \\
\text { "Escasa relación con los apoderados, lo cual, sumado al poco compromiso manifestado por estos, } \\
\text { dificulta la aplicación de estrategias que propicien el desarrollo de hábitos de estudio en los } \\
\text { estudiantes" (E5). } \\
\text { "Dificultad para involucrar a la familia en actividades de escuela que finalmente beneficien a los } \\
\text { alumnos" (E4). } \\
\text { "Dificultad en el desarrollo de estrategias de trabajo colaborativo con los apoderados, para que ellos } \\
\text { potencien el trabajo con sus hijos en casa" (E8). } \\
\text { "Dificultad para desarrollar habilidades y aplicación de solución de conflictos con la familia, por el } \\
\text { escaso conocimiento del contexto sociocultural de los estudiantes y familias" (E18). }\end{array}$ \\
\hline $\begin{array}{l}\text { Manejo actualizado de la profesión, el } \\
\text { sistema educativo y las políticas } \\
\text { vigentes. }\end{array}$ & $\begin{array}{l}\text { "El conocer parcialmente la cultura escolar del establecimiento, me provoca inseguridad y temor a } \\
\text { cometer errores" (E7). } \\
\text { "El conocimiento de los protocolos a seguir en la comunidad educativa me genera inseguridades, } \\
\text { pues pretendo hacer bien las cosas" (E2). } \\
\text { "He notado que cuesta enterarse de lo que pasa en la escuela y al final siempre me entero por } \\
\text { terceras personas, no por los canales oficiales de información" (E12). } \\
\text { "Mucha inseguridad me provocó el desconocimiento respecto de los procedimientos administrativos } \\
\text { del establecimiento, protocolos de actuación, reglamentos internos, Proyecto Educativo Institucional, } \\
\text { plataforma digital de planificaciones y evaluaciones" (E4). } \\
\text { "Dificultad para dar respuesta a las labores administrativas por desconocimiento de políticas } \\
\text { nacionales de la educación relacionadas con el curriculum, la gestión educativa y la profesión } \\
\text { docente" (E9). }\end{array}$ \\
\hline
\end{tabular}


Este constituye un ámbito particularmente problemático para el profesorado principiante. Están las dificultades asociadas a la reflexión sobre la propia práctica, la cual plantean en términos del análisis crítico que realizan de la misma y su reformulación, a partir de los resultados de aprendizaje de los estudiantes. Otro foco de preocupaciones al cual hace frente el novel en este ámbito se vincula con la construcción de relaciones profesionales y de equipo con sus colegas. Este ámbito de desempeño se muestra sensible en cuanto a la capacidad de dialogar con sus pares en aspectos pedagógicos y didácticos. Ampliando el ámbito de las relaciones, un nudo problemático es el desarrollo de relaciones de colaboración y respeto con los padres y apoderados, específicamente al momento de involucrarlos en el aprendizaje y la escuela. Finalmente, también se muestra problemática la tarea de manejar información actualizada sobre su profesión, el sistema educativo y las políticas vigentes, lo que se traduce en preocupaciones asociadas al conocimiento y manejo de las políticas y metas del establecimiento, así como de sus normas de funcionamiento y convivencia.

Desde la perspectiva del desarrollo profesional, este ámbito tensiona el desempeño del principiante por las demandas que representa el trabajo con los pares, la relación con las familias y las condicionantes de la cultura escolar. En este último caso, las características de los contextos de inserción parecen incidir como modeladores de los comportamientos docentes y ofrecen estrategias de vinculación disímiles a los nuevos docentes, desde exigencias muy bajas o inexistentes hasta entornos de fuerte marcación, control y exigencia (Ruffinelli et al., 2017). Además, que este aprendizaje de la profesión se da particularmente en solitario (Ávalos y Valenzuela, 2016) aprendiendo de sus momentos difíciles y movilizando las estrategias que con el tiempo van encontrando para dar respuesta a sus experiencias desafiantes (Çakmak et al., 2019)

Los resultados del estudio son coincidentes con investigaciones nacionales e internacionales respecto a las preocupaciones en torno al espacio del aula (creación de una ambiente propicio de aprendizaje y enseñanza para el aprendizaje de todos los estudiantes), sin embargo, muestran novedad al develar con intensidad un ámbito desconocido y que golpea con fuerza al principiante, vinculado a la cultura escolar y la necesidad de su apropiación (Ferrada, 2019) y que se encuentra complejizado, además, por los requerimientos de la política educacional y por el influjo de la familia (Amin y Rahimi, 2018; Chaaban y Du, 2017). Estos escenarios, ponen de manifiesto también la necesaria colaboración a la hora de asumir una tarea que se torna compleja y desafiante. Enfrenta la cuestión dilemática entre la orientación de la función docente hacia tareas de innovación y mejora de los procesos educativos y la orientación de la acción hacia una configuración más funcional en el contexto de la práctica. Quedan en evidencia las dificultades de la formación del profesorado para avanzar en transformación y no seguir replicando solo continuidad y permanencia.

En consecuencia, las instituciones formadoras de profesores deben ser capaces de reorientar su tarea en torno a una visión más holística, situada y justa de la preparación docente, con un principiante facultado para adaptarse a contextos no solo técnicos sino más bien sociopolíticos (Pardo et al., 2019), capaz de actuar en el marco de relaciones intersubjetivas y de relaciones sociales con los pares y la comunidad que los condicionan y, por sobretodo implican un decidido reconocimiento - expresión de una justicia- al influjo que ejercen las propias interpretaciones, en la forma como se actúa y se es docente (Tardif, 2004; Fantilli, y McDougall, 2009; Ruffinelli, 2014).

Finalmente, las implicancias del estudio permiten sostener la demanda de una formación del profesorado que habilite en unas actuaciones y escenarios cambiantes, reconocer la necesidad de complejizar su discusión atendiendo a los nuevos órdenes sociales y culturales en que tiene lugar el proceso formativo y el trabajo de los profesores, el cual viene a configurar nuevos y más complejos dilemas para los cuales la preparación universitaria parece quedar al debe. Desde la perspectiva del principiante, esta investigación pone de relieve el valor de la reflexión docente en tanto herramienta para enfrentar los desafíos del proceso de inserción (Ruffinelli et al., 2017) e incrementar sus niveles de competencias docente (Goh et al., 2017).

\section{CONCLUSIONES}

La evidencia investigativa muestra que la formación inicial no concluye el objetivo de haber enseñado las competencias básicas de la profesión, sino que éstas se adquieren durante los primeros años de docencia en el contexto de la práctica, y en ese trayecto el proceso de socialización que experimenta el profesorado con los actores (alumnos, profesores, directivos y familia) y el contexto (microcultura y las demandas del sistema social) es el que define los esquemas de actuación profesional. De acuerdo a los resultados de este estudio, se pueden extraer las siguientes conclusiones principales:

Primero, al enfrentarse al ejercicio de la docencia, el profesorado principiante entrevistado experimenta un proceso de aprendizaje que sigue un ciclo en el que la comprensión, enseñanza, evaluación y reflexión de aquello que experimenta le va permitiendo desarrollar una estructura de conocimiento de la profesión, la cual se ve influenciada (facilitada y/u obstaculizada) por las condicionantes sociales que la práctica le impone, en una interacción de aspectos "objetivos" - estándares, fines, metas, valores institucionales, responsabilidad 
social, resultados $-\mathrm{y}$ aspectos subjetivos - autopercepción, expectativas, creencias, preocupaciones y supuestos.

Segundo, en el ejercicio profesional, el profesorado transita de manera diversa entre distintos focos de preocupación. Por una parte, las inseguridades vinculadas a estar haciendo lo que se supone debe hacer, y la adecuación de su comportamiento a las situaciones que enfrenta. Por otra, las preocupaciones vinculadas a los niños, y específicamente su comportamiento, en términos de orden y control para el desarrollo del trabajo docente y la gestión de aula, hasta que emerge un foco con centralidad en el aprendizaje, el cual se ve reflejado por interrogantes del tipo ¿Se están generando aprendizajes?, ¿están aprendiendo todo lo que necesitan aprender?, ¿mi actuación profesional favorece el aprendizaje de todos los estudiantes?, entre otras.

Tercero, en el desarrollo de la docencia, el principiante avanza en la comprensión de la complejidad que encierra su tarea. Transita desde la percepción limitada de las situaciones que experimenta hasta lograr una comprensión más profunda de las mismas que le permite iniciar nuevos desarrollos profesionales.

Cuarto, así el principiante, de acuerdo a los resultados del estudio, parte con una visión centrada en el aula y en la gestión de esa tarea, para luego avanzar a las demandas derivadas del estudiantado y de la diversidad de su perfil que viene a condicionar la planificación de la tarea y su desarrollo. Desde ella, la preocupación se concentra en el aula en términos primero, de asegurar un comportamiento adecuado y segundo, un monitoreo que permita anclar aprendizajes. Prontamente, las preocupaciones, dejan de estar circunscritas al aula, para abrirse a la institución, su cultura y estándares de desempeño; y luego al contexto, y las complejidades que representan las familias. En todo este proceso, las preocupaciones ponen de manifiesto la presencia de una visión fragmentada o parcelada de la naturaleza del trabajo docente, lo que probablemente esté asociada a la presencia de una preparación inicial que no logra familiarizarlo con una actuación flexible y cambiante, y que, al anclarlo a la ilusión de la explicación teórica en su enfrentamiento a la práctica, lo reduce a una actuación parcial y por tanto insuficiente.

Quinto, el ámbito de las responsabilidades profesionales se perfila como el espacio que más tensiona la inserción del profesor principiante, específicamente en lo que significa adaptarse y responder a los requerimientos de una cultura escolar que implica desplegar relaciones profesionales con pares y autoridades; ampliar las continuidades familia - escuela necesarias para el desarrollo de proceso de enseñanza culturalmente sensibles; y que al parecer ponen de manifiesto una falta de correspondencia con los aprendizajes de la formación inicial, obligándolos a iniciar nuevos procesos de aprendizaje de la profesión. Ello abre nuevos espacios de discusión y estudios para que las instituciones formadoras de profesores reactualicen sus procesos formativos; a los establecimientos educacionales para que cuestionen el rol de presión o acompañamiento que están desarrollando; y al Ministerio, para orientar los procesos de mentoría en el contexto de la Ley de Desarrollo Profesional Docente.

\section{REFERENCIAS}

Alliaud, A., Los artesanos de la enseñanza. Acerca de la formación de maestros con oficio, $1^{\circ}$ edición, Paidós, Buenos Aires, Argentina (2017)

Alsaeedi, F. y Male, T., Transformational Leadership and Globalization: Attitudes of School Principals in Kuwait, doi: doi.org/10.1177/1741143213488588, Educational Management Administration \& Leadership, 41(5), 640-657, (2013)

Amin, M., Rahimi, M. y Challenges, A., Faced by Novice EFL Teachers, International Journal of Humanities and Cultural Studies (IJHCS) ISSN 2356-5926, 5(1), 149-166, (2018)

Ávalos, B. y Valenzuela, J., Education for all and Attrition / Retention of new Teachers: A Trajectory Study in Chile, doi: dx.doi.org/10.1016/j.ijedudev.2016.03.012, International Journal of Educational Development, 49, 279-290, (2016)

Ávalos, B., La inserción profesional de los docentes. Profesorado. Revista de currículum y formación del profesorado, ISSN 1138-414X, 13(1), 44-59, (2009)

Bottery, M., Education and Globalization: Redefining the Role of the Educational Professional, doi: doi.org/10.1080/00131910500352804, Educational review, 58(1), 95-113, (2006)

Chaaban, Y. y Du, X., Novice Teachers' job Satisfaction and Coping Strategies: Overcoming Contextual Challenges at Qatari Government Schools, doi: 10.1016/j.tate.2017.07.002, Teaching and Teacher Education, 67, 340-350, (2017)

Çakmak, M., Gündüz, M. y Emstad, A., Challenging Moments of Novice Teachers: Survival Strategies Developed Through Experiences, doi: doi.org/10.1080/0305764X.2018.1476465, Cambridge Journal of Education, 49(2), 147-162, (2019)

Cisternas, T., La Investigación sobre Formación Docente en Chile. Territorios explorados e inexplorados, doi: doi: dx.doi.org/10.31619/caledu.n35.98, Calidad en la Educación, 35, 131-164, (2011)

Fantilli, R. y McDougall, D., A Study of Novice Teachers: Challenges and Supports in the First Years, doi: 10.1016/j.tate.2009.02.021, Teaching and Teacher Education, 25(6), 814-825, (2009) 
Feiman-Nemser, S., Helping Novices Learn to Teach Lessons from an Exemplary Support Teacher, doi: doi.org/ 10.1177/0022487101052001003, Journal of Teacher Education, 52(1), 17-30, (2001)

Ferrada, D., Tuning in the Initial Training of Teachers. A View from Chile, doi: doi.org/ 10.24320/redie.2019.21.e39.2113, Revista Electrónica de Investigación Educativa, 21, 1-14, (2019)

Flick, U., Introducción a la investigación cualitativa, $2^{\circ}$ edición. Morata, Madrid, España (2007)

Goh, P., Yusuf, Q. y Wong, K., Lived Experience: Perceptions of Competency of Novice Teachers, ISSN-1694609X, International Journal of Instruction, 10(1), 21-36, (2017)

Hogan, T., Rabinowitz, M. y Craven, J. A., Representation in teaching: Inferences from research of expert and novice teachers, doi: doi.org/10.1207/S15326985EP3804_3, Educational Psychologist, 38(4), 235-247, (2010)

Kim, H. y Cho, Y., Pre-service Teachers' Motivation, sense of Teaching Efficacy, and Expectation of Reality Shock, doi: dx.doi.org/10.1080 / 1359866X.2013.855999, Asia-Pacific Journal of Teacher Education, 42(1), 67-81, (2014)

Pardo, N., Rodriguez, D. y Dallos, Y., Prácticas Educativas de los Educadores Piapoco en torno a la Construcción de Escenarios de Paz en Comunidades del Departamento de Guainía en Colombia, doi: dx.doi.org/10.4067/S071807642019000500203, Información Tecnológica, 30(5), 203-212 (2019)

Ruffinelli, A., Cisternas, T. y Córdoba, C., Iniciarse en la docencia. ISBN 978-956-357-105-9, Ediciones Universidad Alberto Hurtado, Santiago, Chile, (2017)

Ruffinelli, A., ¿Qué aprenden los docentes en su primer año de ejercicio profesional?: representaciones de los propios docentes principiantes, doi: dx.doi.org//10.7764/PEL.51.2.2014.5, Pensamiento Educativo. Revista de Investigación Educacional Latinoamericana, 51(2), 56-74, (2014).

Ruiz, J., Metodología de la investigación cualitativa, 3 edición, ISBN: 84-7485-423-7, Universidad de Deusto, Bilbao, España (2009)

Solís, M., Nuñez, C., Contreras, I., Vásquez, N. y Ritterhaussen, S., Inserción Profesional Docente: problemas y éxitos de los Profesores Principiantes, Estudios Pedagógicos, doi: dx.doi.org/10.4067/S0718-07052016000200019, 42 (2) 331-342, (2016)

Tardif, M., Los saberes del docente y su desarrollo profesional, ISBN 84-217-1450-5, Narcea, Madrid, España (2004) 